\title{
Estudio piloto de consulta telefónica pediátrica
}

\author{
MC. García de Ribera ${ }^{\mathrm{a}}$, ME. Vázquez Fernández \\ MR. Bachiller Luquec, MP. Barrio Alonso ${ }^{d}$, MF. Muñoz Moreno ${ }^{e}$, \\ J. Posadas Alonsof, AM. Hernández Vázquez ${ }^{\S}$, P. Hernández Velázquez ${ }^{h}$ \\ 'MIR-Pediatría, Hospital Clínico Universitario. Valladolid. España.

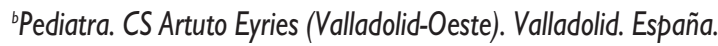 \\ 'Pediatra. CS Pilarica (Valladolid-Este). Valladolid. España. \\ ¿Pediatra de Área (Valladolid Oeste). Valladolid. España. \\ eUnidad de Apoyo a la Investigación. Hospital Clínico Universitario. Valladolid. España. \\ fCoordinador de Equipos, GAP del Área Valladolid-Este. Valladolid. España. \\ ${ }^{\circledR}$ Enfermera de Pediatría. CS Barrio España (Valladolid-Oeste). Valladolid. España. \\ ${ }^{h}$ Enfermera de Pediatría. CS Gamazo (Valladolid-Oeste). Valladolid. España.
}

\section{Resumen}

Introducción. Las urgencias pediátricas en Atención Primaria han aumentado enormemente en los últimos años. Los motivos de consulta suelen ser procesos banales, cuadros autolimitados que saturan las urgencias y obstaculizan la atención del niño críticamente enfermo.

Presentamos un estudio piloto en población pediátrica de un centro de salud urbano, tras la implementación de consulta telefónica durante 6 meses.

Material y métodos. Se realizó un estudio ambispectivo con una fase prospectiva a lo largo de seis meses y un análisis retrospectivo de la información generada. Se incluyeron como sujetos todos aquellos que solicitaban demanda telefónica de consulta. En cada uno de ellos se monitorizaron variables demográficas, socio-sanitarias, epidemiológicas y clínicas.

Resultados. Se registraron 419 consultas telefónicas. El 37\% fueron atendidas por residentes, $33 \%$ por pediatras y $30 \%$ por enfermeras dedicadas a pediatría. Los menores de 2 años supusieron el $48 \%$ de las consultas atendidas y no hubo diferencias entre sexos. Los motivos más frecuentes de consulta fueron síntomas gastrointestinales y fiebre. En las 24 horas siguientes se comprobó que el consejo fue resolutivo en el 56\%, 27\% acudió a su pediatra, 15\% necesitaron acudir al servicio de urgencias y solo el $1 \%$ precisó ingreso. Se obtuvo una puntuación media de satisfacción de 9,24.

Conclusiones. Destacamos que la consulta telefónica resuelve adecuadamente la mayoría de los problemas de salud planteados y que proporciona niveles muy altos de satisfacción de la población atendida.

Palabras clave: Consulta telefónica. Urgencias. Cribado.

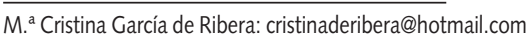

Los autores declaran no presentar conflictos de intereses en relación con la preparación y publicación de este artículo. 
Pilot study of a telephone paediatric consultation

\section{Abstract}

Introduction. Paediatric emergencies in primary care have increased dramatically in the last years. The chief complaints are often mild diseases that clutters the casualty wards and obstruct critical child care.

We present a pilot study in the paediatric population of an urban health centre, after the implementation of the telephone consultation for 6 months.

Material and methods. The study was performed with a prospective phase over six months and a retrospective analysis of the information generated. We included as subjects all those who requested telephone consultation. In each of these people were collected demographic, socio-sanitary, epidemiological and clinical variables.

Results. A total of 419 telephone consultations were managed, $37 \%$ were attended by residents, $33 \%$ by paediatricians and $30 \%$ by paediatric nurses. Forty-eight percent of the handled cases were children under 2 years, and there were no differences between sexes. The most frequent reasons for consultation were gastrointestinal symptoms and fever. Within the next 24 hours, it was found that the consultation was resolved in $56 \%$ of the cases, $27 \%$ went to their paediatrician, $15 \%$ needed to go to the emergency room and only $1 \%$ required admission. It showed an average satisfaction of 9.24 .

Conclusions. The conclusions highlight that telephone consultation address adequately most health problems encountered and provides very high levels of satisfaction among the population served.

Key words: Telephone consultation. Emergency. Screening.

\section{Introducción}

En los últimos años, las visitas a los Servicios de Urgencias de Pediatría, tanto de Atención Primaria (AP) como hospitalarios, han experimentado un ascenso importante. La mayoría de las patologías que motivan estas visitas al especialista son de características banales, y muchas veces sirven para tranquilizar a los padres más que para sanar al hijo ${ }^{1}$.

Se plantean muchos interrogantes a nivel de la gestión de la salud en torno a qué medidas serían más eficaces para reducir el uso masivo de estos servicios y para establecer unos sistemas de selección que determinen la prioridad de la urgencia. La consulta telefónica pediátrica se puede encuadrar dentro de estas medidas, por la fácil accesibilidad a nivel de la población y la creciente experiencia de uso existente en estos momentos entre los profesionales sanitarios. La inmediatez de respuesta ante la demanda del paciente, junto con la seguridad y fiabilidad avalada por distintos estudios, se plantea como la mayor ventaja a la hora de intentar aliviar la carga asistencial de las Urgencias Pediátricas ${ }^{2-4}$.

En Estados Unidos existen centros de llamadas, con personal entrenado y remunerado (en su mayoría enfermeras pediátricas), que atienden la demanda tele- 
fónica en el horario que excede la jornada laboral del pediatra ${ }^{5}$. En España se han estudiado las consultas telefónicas recibidas en centros de $\mathrm{AP}^{6} \mathrm{y}$ se han ensayado protocolos de triage telefónico atendidos por los residentes de Pediatría desde el Servicio de Urgencias hospitalario?.

En nuestro estudio, presentamos la implementación de un servicio de consulta telefónica realizado fuera del horario laboral, de 15:00 a 08:00 horas, en un centro de salud urbano de Valladolid de enero a junio de 2009.

Como objetivos, pretendemos analizar las características clínico-epidemiológicas del usuario, la capacidad de resolución de la problemática planteada y los índices de satisfacción del usuario, y evaluar las diferencias en la actuación de los diferentes profesionales que atienden las llamadas: pediatras, enfermeras y residentes (MIR).

\section{Material y métodos}

El presente trabajo se concibe como un estudio ambispectivo, con una fase prospectiva de recogida de datos a lo largo de seis meses y un análisis retrospectivo de la información generada.

Para llevarlo a cabo, se formó un equipo de trabajo integrado por profesionales sanitarios con experiencia en el ámbito de la Pediatría: dos enfermeras, dos pedia- tras y dos residentes de tercer y cuarto año. Se llevó a cabo un programa de formación en los meses previos al comienzo de la puesta en marcha del teléfono, creando protocolos específicos para la atención de la patología más demandada en los Servicios de Urgencias, en los que se hacía especial hincapié en los síntomas de alarma, así como un entrenamiento tendente a minimizar la variabilidad de los profesionales que atienden la consulta.

Por ello se contrató un único número de teléfono, proporcionando un terminal a cada profesional para poder atender las guardias telefónicas. Este servicio telefónico se publicitó a los pacientes pediátricos del centro de salud PilaricaCircular mediante carta personalizada y carteles informativos en el centro. El proyecto comenzó a funcionar el 8 de enero de 2009, con horario de lunes a viernes, de 15:00 a 08:00 horas (h), y los fines de semana, de 08:00 h a 08:00 h, y finalizó el 30 de junio de 2009.

En el momento de cada llamada se cumplimentó un protocolo con el que se confeccionó una base de datos que recogía:

- Datos sociodemográficos del niño: edad, sexo, domicilio, trabajo de los padres, nacionalidad, número de hermanos y asistencia a colegio o guardería. 
- Presencia de enfermedades crónicas 0 alergias.

- Motivo de consulta.

- Sintomatología que presentaba: fiebre, vómitos, diarrea, granos o manchas, tos, mocos, dolor de oídos o sospecha de intoxicación o accidente.

- Otros motivos de consulta distintos a procesos patológicos como: dudas sobre fármacos, de puericultura o del funcionamiento de sistema sanitario.

- Consejo aportado por el profesional que contestó la llamada: sanitario, de puericultura o derivación a Urgencias (al Punto de Atención Continuada [PAC], o al hospital).

- Categoría profesional que atiende la llamada: médico, enfermera o residente de Pediatría.

- Horario y día de la semana de atención de la llamada.

A las 24 horas se contactó telefónicamente con cada paciente, recogiendo la evolución del mismo, la necesidad de acudir a otro servicio y el nivel de satisfacción del tutor que realizó la consulta telefónica.

El programa estadístico utilizado para el análisis ha sido SPSS ${ }^{\circledR}$ 17.0. Se realizó estadística descriptiva e inferencial con tablas de contingencia y se calculó el porcentaje de cada uno de los paráme- tros analizados y el intervalo de confianza del 95\% (IC 95\%). Tras la aplicación de los test de normalidad de Kolmogorov-Smirnov y de Shapiro-Wilk, los resultados se compararon mediante los test de Kruskal Wallis y Chi cuadrado, considerándose diferencias significativas cuando el valor de $p<0,05$.

\section{Resultados}

Durante los seis meses en que se llevó a cabo el proyecto se atendieron 419 consultas telefónicas con una media diaria de 2,34 llamadas, lo que supone el $5,8 \%$ del total de consultas atendidas en nuestro PAC (7249 consultas durante el mismo periodo).

No se observan diferencias significativas en frecuencia de llamadas entre niños y niñas, siendo varones un $51 \%$ y mujeres un $49 \%$. La edad media de los que consultaron fue 38 meses (IC 95\%: 34-41 meses). El 48\% de las llamadas fueron relacionadas con pacientes entre 0 y 23 meses. En la figura 1 se puede ver la distribución por edades.

Por centros de salud: observamos que en el de mayor nivel socioeconómico, con una población media de 958 niños atendidos por un solo pediatra, la consulta telefónica supone el $36 \%$ y en el de menor nivel socioeconómico, con una población media de 1957 niños 
Figura 1. Distribución por edades.

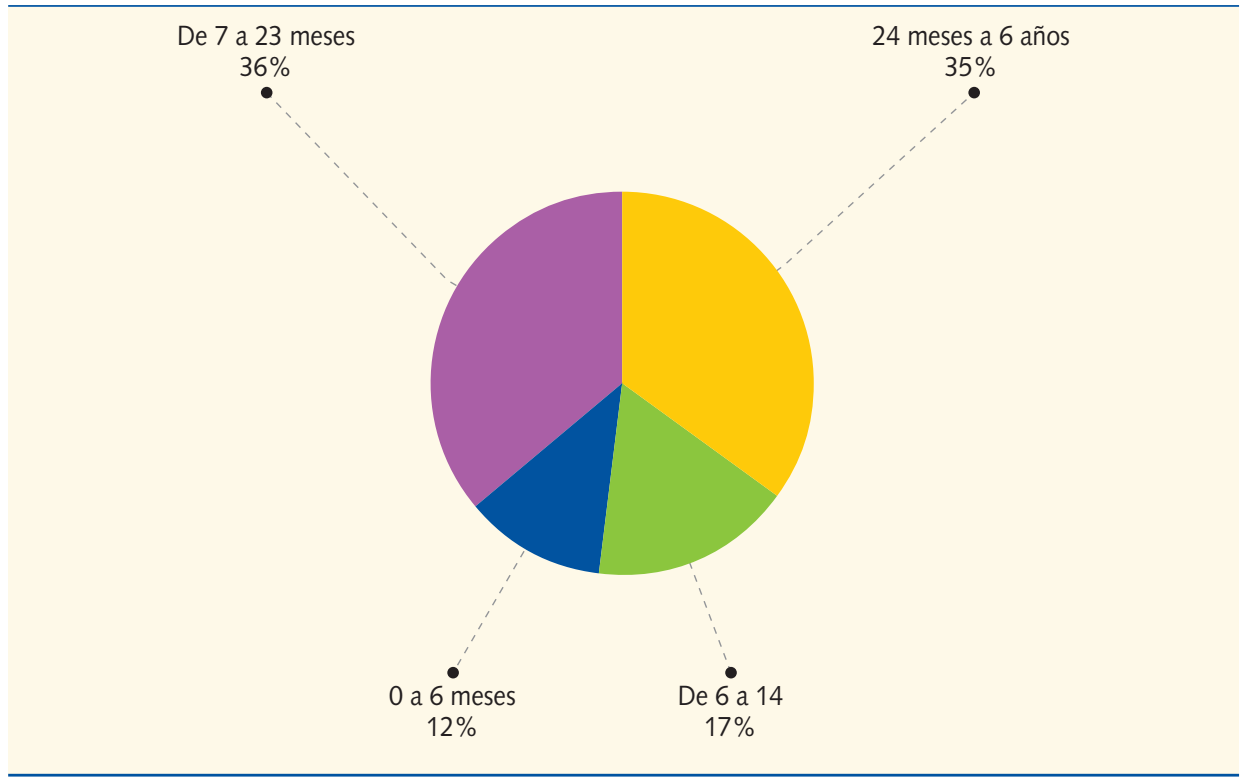

atendidos por dos pediatras, el $59 \%$, sin diferencias significativas. En el $5 \%$ de las llamadas al usuario le correspondía otro centro de salud.

El $52 \%$ de los pacientes no tenían hermano, el $33 \%$ tenían un hermano y solo un $5 \%$ eran familia numerosa. El $63 \%$ acudían a colegio o guardería. El $95 \%$ de los padres eran de procedencia española, solo un $5 \%$ eran inmigrantes, con procedencia diversa.

La mayoría de los pacientes que utilizaron el servicio $(95 \%)$ no presentaba ningún tipo de enfermedad crónica y solo el $2 \%$ refieren alergia a medicamentos.

En la figura 2 se observa la distribución del motivo principal de consulta telefó- nica. El más frecuente fue la consulta por síntomas, y, dentro de él, la fiebre y la sintomatología digestiva, seguidas de síntomas otorrinolaringológicos (ORL). No se registró ninguna consulta sobre patología de riesgo vital.

Cuando al usuario se le pregunta por los síntomas que presenta, encontramos que lo más habitual es la mucosidad, en casi el $40 \%$ de los pacientes, seguida muy de cerca por la tos y la fiebre. Con porcentajes similares estarían los vómitos y la diarrea (18\% cada uno).

En 234 llamadas se registraron otros tipos de consulta generalmente asociadas a las consultas por los problemas de 
Figura 2. Motivos de consulta.

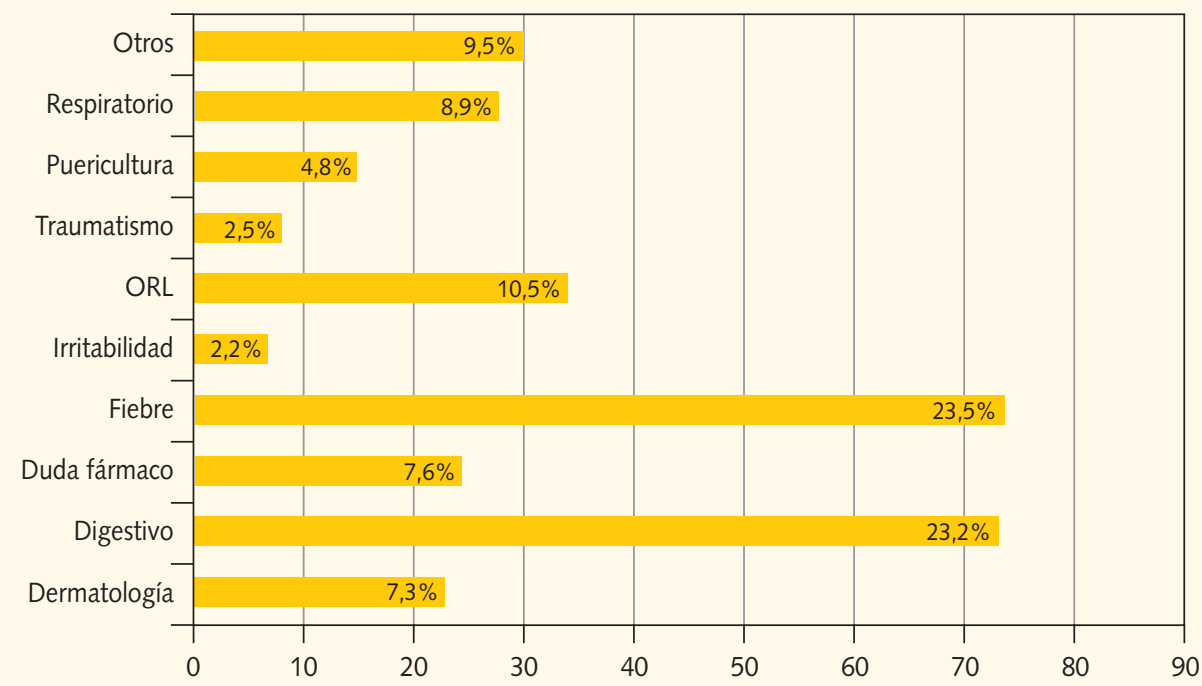

salud ya comentados: $47 \%$ información de puericultura, $45 \%$ dudas de fárma$\cos , 5 \%$ información sobre funcionamiento del sistema sanitario y $3 \%$ consulta administrativa.

En la mayoría de los casos recibieron instrucciones sanitarias para el manejo domiciliario o se los instó a acudir a su pediatra en caso de empeorar. A un 9,5\% se le recomendó acudir al PAC y a algo menos del $5 \%$ al Servicio de Urgencias hospitalario.

A las 24 horas, mediante nuevo contacto telefónico realizado por el mismo profesional que atendió la consulta, se comprobó que la mayoría (77\%) había experimentado mejoría. Tan solo un 4\% refiere un empeoramiento del cuadro. El resto se mantuvo estable. Un $56 \%$ de los atendidos por nuestro servicio no precisó atención sanitaria posterior. En torno a un $28 \%$ visitó a su pediatra y un 15\% visitó los Servicios de Urgencia del PAC o del hospital, precisando ingreso hospitalario cuatro niños (el $1 \%$ de los atendidos). El nivel de satisfacción declarado ha sido muy alto. Sobre una escala de 0 a 10 se obtuvo una puntuación media de 9,24 y ninguna por debajo de 5 .

El profesional que atendió y resolvió la llamada fue el pediatra en el $33 \%$ de las llamadas, el residente en un $37 \%$ y la en- 
Tabla 1. Derivación por titulación profesional; derivación vs. titulación de la persona que atiende $(P<0,001)$

\begin{tabular}{|c|c|c|c|c|c|c|}
\hline & \multicolumn{4}{|c|}{ Titulación de la persona que atiende } \\
\hline & & & Enfermería & MIR & Médico & Total \\
\hline \multirow[t]{14}{*}{ Derivación } & \multirow[t]{2}{*}{ No necesitó } & $\mathrm{n}$ & 44 & 78 & 47 & 169 \\
\hline & & $\%$ & $52,4 \%$ & $58,2 \%$ & $56,6 \%$ & $56,1 \%$ \\
\hline & \multirow[t]{2}{*}{ Urgencias PAC } & $\mathrm{n}$ & 6 & 12 & 4 & 22 \\
\hline & & $\%$ & $7,1 \%$ & $9,0 \%$ & $4,8 \%$ & $7,3 \%$ \\
\hline & \multirow[t]{2}{*}{ Pediatra } & $\mathrm{n}$ & 33 & 22 & 28 & 83 \\
\hline & & $\%$ & $39,3 \%$ & $16,4 \%$ & $33,7 \%$ & $27,6 \%$ \\
\hline & \multirow[t]{2}{*}{ Urgencia hospitalaria } & $\mathrm{n}$ & 1 & 17 & 4 & 22 \\
\hline & & $\%$ & $1,2 \%$ & $12,7 \%$ & $4,8 \%$ & $7,3 \%$ \\
\hline & \multirow[t]{2}{*}{ Ingreso hospitalario } & $\mathrm{n}$ & 0 & 4 & 0 & 4 \\
\hline & & $\%$ & $0,0 \%$ & $3,0 \%$ & $0,0 \%$ & $1,3 \%$ \\
\hline & \multirow[t]{2}{*}{$\begin{array}{l}\text { Pediatra + Urgencia } \\
\text { hospitalaria }\end{array}$} & $\mathrm{n}$ & 0 & 1 & 0 & 1 \\
\hline & & $\%$ & $0,0 \%$ & $0,7 \%$ & $0,0 \%$ & $0,3 \%$ \\
\hline & \multirow[t]{2}{*}{ Total } & $n$ & 84 & 134 & 83 & 301 \\
\hline & & $\%$ & $100,0 \%$ & $100,0 \%$ & $100,0 \%$ & $100,0 \%$ \\
\hline
\end{tabular}

Figura 3. Volumen (número) de llamadas por días de la semana en el periodo de tiempo estudiado.

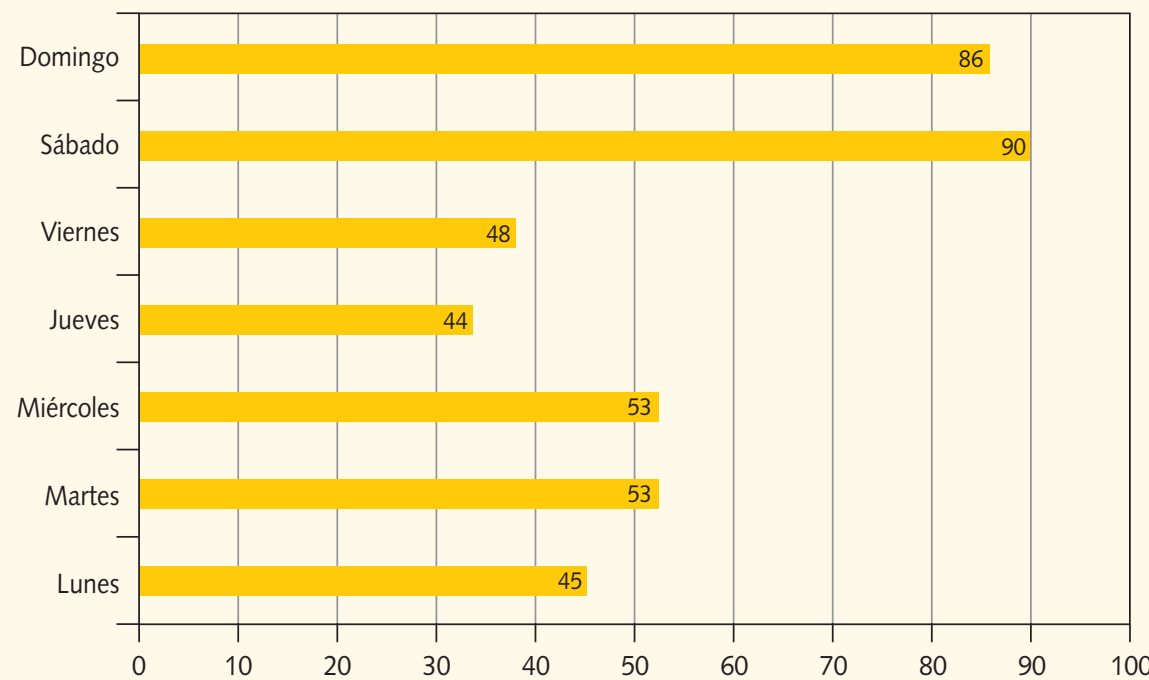


fermera en un $30 \%$. Hemos separado las llamadas atendidas por cada categoría profesional y comparado entre ellos según las actitudes que se tomaron a consecuencia de la llamada. Encontramos mayor número de llamadas atendidas por el residente que precisaron derivación, obteniendo diferencias significativas en la capacidad resolutiva de cada tipo de trabajador, como se ve en la tabla 1.

Se observa un alto volumen de demanda los meses de invierno, con descenso progresivo en los de primavera. El porcentaje más alto se registró en febrero $(21 \%)$ y el más bajo en mayo $(6 \%)$. Observándolo por días (figura 3), el mayor número de llamadas se produjo durante el fin de semana. Los sábados suponen el $21,5 \%$ del total, aunque sin diferencias significativas.

Tanto en días laborables como festivos el horario más amplio de atención ha sido de 15:00 a 22:30 horas, con el 63\% de frecuentación. Hay que recordar que el horario de mañana (de 08:00 a 15:00 horas) se realizó solo los sábados y domingos. La noche, de 22:30 a 08:00 h, fue menos usual, con el $18 \%$ del volumen de las llamadas.

\section{Discusión y conclusiones}

La saturación creciente de los Servicios de Urgencia pediátricos, debido en parte a la utilización inapropiada de estos por niños con patología banal, cuestiona los sistemas actuales de sanidad ${ }^{8}$. Por ello, se plantean nuevas medidas educacionales de la población y recursos que hagan posible la resolución de los problemas que preocupan a los progenitores actuales, de forma más efectiva.

La consulta telefónica entraría a formar parte de estas nuevas medidas. Por lo general, este sistema ayuda, orienta, asesora, proporciona información y permite realizar triage de casos que necesitan atención médica urgente 3 . Incluso algunos programas de formación de residentes en distintos países incluyen un entrenamiento en el manejo de las Ilamadas telefónicas?.

Hasta la fecha hay relativamente poca información sobre si la consulta telefónica reduce la asistencia a otros servicios médicos. En Dinamarca, después de cinco años de funcionamiento, las visitas a domicilio se redujeron un $28 \%{ }^{10}$. En el Reino Unido hubo una pequeña disminución en la atención urgente de los médicos generales $^{11}$. Sin embargo, otros opinan que el teléfono tiene una capacidad limitada para mejorar el uso de Servicios de Urgencia pediátricos ${ }^{12,13}$. Pocos estudios informaron sobre efectos adversos.

En el trabajo que presentamos, el volumen de llamadas recibidas correspon- 
dientes a dos centros de salud supone un $5,8 \%$ del total de consultas atendidas en nuestro PAC, que atiende las urgencias de toda el Área de Salud, lo que supone un importante volumen relativo de las urgencias atendidas. De todos modos, observamos que un pequeño porcentaje $(5 \%)$ de las llamadas procedían de pacientes adscritos a otros centros de salud del área sanitaria, efecto de la buena acogida del servicio y del efecto divulgativo por parte de los usuarios.

En cuanto a las características de los usuarios de la consulta telefónica, los resultados son muy similares a los de otras series $^{6,7}$. Hay un alto porcentaje (48\%) de menores de dos años, coincidiendo con la edad de los mayores frecuentadores de las Urgencias pediátricas ${ }^{14}$. Más de la mitad de los pacientes eran hijos únicos, de acuerdo con la media poblacional. Estos dos datos aportan las características del perfil de usuario del servicio telefónico más frecuente: padres de primer hijo, en época lactante. Tan solo un $5 \%$ de los que llamaron tenían tres 0 más hijos, lo que se puede explicar considerando la menor frecuencia de estas familias a nivel poblacional, sumando el hecho de la menor angustia ante las enfermedades que aporta tener experiencia con los otros hijos. La mayoría (95\%) eran de nacionalidad española, siendo difícil extrapolarlo a datos poblacionales, ya que muchos de los extranjeros no tienen su documentación en regla, pero sí es de tener en cuenta sus dificultades con el idioma, por lo que les resulta más difícil el uso del teléfono, y su menor nivel económico, que limita el acceso a esta línea no gratuita. En algún trabajo también se ha argumentado que las minorías étnicas y grupos desfavorecidos infrautilizan el servicio ${ }^{15}$.

En patologías crónicas, la posibilidad de consejo telefónico permanente es útil para responder de forma adecuada a las necesidades de los pacientes y sus familias $^{16,17}$. En nuestro trabajo solo un 5\% refería enfermedad crónica. Hay que interpretar este dato considerando el relativo fácil acceso que tienen estos niños (fibróticos quísticos, oncológicos, neurológicos, etc.) vía telefónica en nuestra área de salud, con la posibilidad de consultar frecuentemente a su especialista. También se recogió el dato de alergias a medicamentos, por el peligro que entraña una posible reacción anafiláctica en el domicilio, refiriendo este riesgo solo un $2 \%$.

El motivo principal de consulta era la presencia de síntomas, seguido a mucha distancia por las consultas de puericultura y dudas sobre fármacos. Los síntomas que referían eran en su mayoría leves: mocos, tos y fiebre. 
En la consulta telefónica existen varias cuestiones a las que se puede responder con facilidad: dosis y efectos de fárma$\cos$, dudas de puericultura, actitud ante determinadas enfermedades conocidas, pero ante la presencia de síntomas, que es lo habitual, nuestra actitud fue la de orientar a la familia sobre los que deberían ser llevados a valoración médica, inmediata o diferida. Para ello en el periodo de formación de los sanitarios se hizo especial hincapié en los síntomas de alarma susceptibles de derivación hospitalaria, sobre todo la fiebre, para que se vigilara si era elevada, si se acompañaba de exantema y si se presentaba en menores de seis meses. En nuestro estudio tuvimos un porcentaje muy pequeño (11\%) de niños de esta franja de edad que los presentaban.

A las 24 horas de la llamada, el sanitario que les atendió contactaba con ellos telefónicamente, lo que sirvió para evaluar la satisfacción con el servicio prestado y la evolución del niño. Nuestros resultados coinciden con los de otras series; más de la mitad de las consultas pueden ser resueltas mediante instrucciones domiciliarias ${ }^{6,7,18}$. Tan solo a un $15 \%$ se le aconsejó acudir a un Servicio de Urgencias pediátrico. El resto, un $28 \%$, consultó con su pediatra el día siguiente. Por tanto, se puede afirmar que mediante el teléfono se seleccionan pacientes y se evitan visitas innecesarias, si bien esta afirmación puede estar sesgada por el hecho de que los que llamen presenten patologías menos graves que los que acuden a Urgencias, aunque el folleto divulgativo enviado a los padres aconsejaba utilizar el servicio telefónico antes de acudir a Urgencias. El hecho de que un $95 \%$ mejorase o se mantuviera estable confirma la levedad de los pacientes que utilizan este servicio e indica el presumible correcto manejo de su situación del personal que se ocupó de ellos. El elevado nivel de satisfacción se puede explicar en parte por la accesibilidad de este servicio, pudiendo calmar con una conversación la angustia que presentan muchos padres ante el menor síntoma de su primer hijo y en parte por la novedad que representa en nuestra población, que no dispone de estos recursos habitualmente. En otros países que disponen de cobertura telefónica hace años, como Reino Unido, por ejemplo, el nivel de satisfacción con el servicio telefónico, que lleva años implantado, no es tan alto $^{19}$.

Al igual que han demostrado otros estudios $^{20}$ nuestros resultados demuestran que la capacidad resolutiva de los tres tipos de sanitarios que atendieron la llamada ha sido similar, lo que indica la ca- 
pacidad para implementar este servicio que tienen los residentes y enfermeras de pediatría, capacitados previamente con un entrenamiento sencillo en atención telefónica. Uno de los sistemas más grandes de consulta telefónica en funcionamiento es "NHS Direct" en Reino Unido, que está atendido solo por enfermeras $^{21}$.

Respecto al volumen de llamadas, observamos un mayor número de ellas en los meses de invierno y en horario de tarde, coincidiendo con los datos de asistencia a las Urgencias de pediatría ${ }^{22,23}$. Una limitación de nuestro estudio es el no tener datos de un año entero, pero la evolución de la frecuentación en los seis meses que se realizó hace predecir el curso habitual a lo largo de 12 meses.

Con respecto al problema legal que supone la atención médica telefónica, en la actualidad no existe nada en este sentido en nuestro país. Para minimizar este riesgo, en nuestro proyecto se recogie- ron datos epidemiológicos del paciente, los datos de consulta fueron grabados $y$, formando parte del protocolo, se aconsejó acudir a Urgencias en caso de empeorar.

\section{Conclusiones}

Nuestro proyecto demuestra resultados eficientes y satisfactorios para el usuario y la factibilidad del sistema propuesto. Creemos que la inclusión de su uso como un complemento reglado dentro de la práctica clínica puede proporcionar un alto grado de rendimiento como triage para discernir qué casos necesitan atención médica urgente y un método de intervención eficaz, sencillo, cómodo, rápido y barato. En esta línea se plantea una segunda fase del estudio, que amplíe la población a la que atender. A la atención telefónica se podría añadir nuevos métodos de comunicación, empleando, por ejemplo, fotografías, vídeo o Internet.

\section{Bibliografía}

1. Williams A, O'Rourke P, Keogh S. Making choices: why parents present to the emergency department for non-urgent care. Arch Dis Child. 2009;94:817-20.

2. Al-Abdullah $T$, Plint AC, Shaw $A$, Correll $R$, Gaboury I, Pitters $C$ et al. The appropriateness of referrals to a pediatric emergency department via a telephone health line. CJEM. 2009;11:139-48.

3. Bunn F, Byrne G, Kendall S. Telephone consultation and triage: Effects on health care use and patient satisfaction. Cochrane Database Syst Rev. 2004;(4):CD004180.

4. Kempe A, Bunik M, Ellis J, Magid D, Hegarty $T$, Dickinson $L M$ et al. How safe is triage by an af- 
ter-hours telephone call center? Pediatrics. 2006; 118:457-63.

5. Lee TJ, Guzy J, Johnson D, Woo H, Baraff LJ. Caller satisfaction with after-hours telephone advice: nurse advice service versus on-call pediatricians. Pediatrics. 2002;110:865-72.

6. Alberola López $S$, del Real Llorente M, Ortega García R, Maestro González B, Andrés de Llano J. La consulta telefónica: Utilización y posibilidades en atención primaria. Bol Pediatr. 1997;37:221-5.

7. Fernández Landaluce $A$, Andrés Olaizola $A$, Mora González E, Azcunaga Santibañez B, Mintegui Raso S, Benito Fernández J. Triage telefónico realizado por médicos en urgencias de pediatría. An Pediatr (Barc). 2005;63:314-20.

8. Carret ML, Fassa AC, Domingues MR. Inappropriate use of emergency services: a systematic review of prevalence and associated factors. Cad Saude Publica. 2009;25:7-28.

9. Wood PR. Pediatric resident training in telephone management: a survey of training programs in the United States. Pediatrics. 1986;77:822-5.

10. Christensen MB, Olesen F. Out of hours service in Denmark: evaluation five years after reform. BMJ. 1998;316:1502-5.

11. Munro J, Nicholl J, O'Cathain A, Knowles E. Impact of NHS direct on demand for immediate care: observational study. BMJ. 2000;321: 150-3.

12. Graber DJ, Ardagh MW, O'Donovan P, St George I. A telephone advice line does not decrease the number of presentations to Christchurch Emergency Department, but does decrease the number of phone callers seeking advice. N Z Med J. 2003;116:U495.

13. Sprivulis P, Carey M, Rouse I. Compliance with advice and appropriateness of emergency presentation following contact with the HealthDirect telephone triage service. Emerg Med Australas. 2004;16:35-40.
14. Loscertales Abril M, Alonso Salas M, Cano Franco J, Charlo Molina T. Importancia de la clasificación en la atención del niño que acude a una consulta de urgencias. Vox Paediatrica. 2004;12: 7-14.

15. George S. NHS Direct audited. BMJ. 2002; 324:558-9.

16. Coma E, de la Haba I, Comabella R, Zayas $S$, Armisen A, Ciurana R y cols. La atención telefónica urgente especializada: una manera de mejora en la utilización de los servicios de urgencias hospitalarios. Emergencias. 2009;21:117-20.

17. Herreros $O$, Sánchez F, Herrera I, Sáez J, Gracia R, Ferrer Roca O. Telemedicina: intervención telefónica en paidopsiquiatría. Rev Psiquiatr Infant Juv. 2002;1:11-3.

18. Lattimer V, George $S$, Thompson F, Thomas E, Mullee M, Turnbull J et al. Safety and effectiveness of nurse telephone consultation in out of hours primary care: randomised controlled trial. The South Wiltshire Out of Hours Project (SWOOP) Group. BMJ. 1998;317:1054-9.

19. O'Cathain A, Munro JF, Nicholl JP, Knowles E. How helpful is NHS direct? Postal survey of callers. BMJ. 2000;320:1035.

20. Benjamin JT. Pediatric residents' telephone triage experience. Relevant to general pediatric practice? Arch Pediatr Adolesc Med. 1997;151: 1254-7.

21. Calman K. Developing Emergency Services in the Community. The Final Report. London: NHS Executive; 1997.

22. Muñoz García JL, Fandiño Orgeira JM, Díaz Peromingo JA. Demanda asistencial de urgencias pediátricas atendidas en un hospital comarcal. Aten Primaria. 2008:40:297-301.

23. Proctor JH, Hirshberg AJ, Kazzi AA, Parker RB. Providing telephone advice from the emergency department. Ann Emerg Med. 2002;40: 217-9. 